\title{
Relationship between tourism demand in the Swiss Alps and hot summer air temperatures associated with climate change
}

\author{
G. Serquet $\cdot$ M. Rebetez
}

Received: 12 May 2009 / Accepted: 14 October 2010 / Published online: 22 February 2011

(C) Springer Science+Business Media B.V. 2011

\begin{abstract}
We quantified the impacts of hot summer air temperatures on tourism in the Swiss Alps by analysing the relationship between temperature and overnight stays in 40 Alpine resorts. Several temperature thresholds were tested to detect the relationship between them and summer tourism. Our results reveal significant correlations between the number of nights spent in mountain resorts and hot temperatures at lower elevations. The relationship between hot temperatures and overnight stays is more important in June and to a lesser extent in August than in July. This is probably because holidays and the peak of domestic tourist demand in summer usually take place between the beginning of July and mid-August so that long-term planned stays dominate more during these months compared to June. The alpine resorts nearest to cities are most affected by hot temperatures. This is probably because reactions to hot episodes take place on a short-term basis as heat waves remain relatively rare. Our results suggest that alpine tourist resorts could benefit from hotter temperatures at lower elevations under future climates. Tourists already react on a short-term basis to hot days and spend more nights in hotels in mountain resorts. If heat waves become more regular, it seems likely that tourists will choose to stay at alpine resorts more frequently and for longer periods.
\end{abstract}

\section{Introduction}

The Swiss Alps are a traditional destination for tourism, an important component of the Swiss economy. Tourism ranked fourth in the Swiss national export balance in 2006, with a 6.2\% share of gross domestic product (GDP) (STV-FST 2008). Of a total revenue of $\mathrm{CHF} 21.6$ billion for the tourism sector in $2004,44.9 \%$ originated from

G. Serquet · M. Rebetez $(\bowtie)$

WSL Swiss Federal Research Institute, Lausanne, Switzerland e-mail: martine.rebetez@wsl.ch 
domestic tourism (STV-FST 2008). Switzerland's tourism competitiveness ranks 1st among 133 countries (WEF 2007, 2008, 2009), meaning that it is considered to have the most attractive environment for developing the travel and tourism industry. Austria and Germany, two other Alpine countries rank 2nd and 3rd in these reports.

Tourism is the key growth sector in mountain areas: tourism intensity (number of tourists' overnight stays compared to the number of residents) in Switzerland is highest in three administrative Alpine regions: Graubunden, Valais and the Bernese Oberland (OFS 2007). Tourism accounted for 30\% of GDP and 30\% of total employment in Graubunden (25\% and 30\% in Valais) in 2007 (STV-FST 2008).

All IPCC scenarios (IPCC 2007a) predict a decrease in snow cover, particularly at lower altitude, in connection with temperature increases. Many alpine resorts are thus expected to become less reliable for snow activities in the future (Elsasser and Buerki 2002; Elsasser and Messerli 2001; Hantel and Hirtl-Wielke 2007; Koenig and Abegg 1997; Marty 2008; OcCC-ProClim 2007; Scherrer and Appenzeller 2006; Wielke et al. 2004). The potential annual costs of climate change in Switzerland, taking into account losses in winter tourism, have been calculated to be CHF 1.8 to 2.3 billion (US $\$ 1.1$ to 1.4 billion) by 2050 , i.e. $0.3 \%$ to $0.4 \%$ of the Swiss GDP for 1995 (Meier 1998). Alpine ski resorts will thus have to plan and implement adaptation strategies if they are to survive (Elsasser and Buerki 2002; Elsasser and Messerli 2001). One of these strategies could be to make better use of the summer season.

Numerous studies have examined the recent increase in summer heat waves in Europe, particularly after the heat waves of 2003 and 2006 (Della-Marta et al. 2007a, b; Rebetez 2004; Rebetez et al. 2006; Schaer et al. 2004; Seneviratne et al. 2006; Stott et al. 2004), and the climate models associated with all the IPCC scenarios (IPCC 2007a) predict hotter summer temperatures and more heat waves in the future. In connection with climate change, several studies (Amelung and Viner 2006; Ceron and Dubois 2000; Hamilton et al. 2005; IPCC 2007b) predict a gradual shift of tourist destinations further north and to higher altitudes in Europe, but no study has yet been conducted to verify if hot summer temperatures generate an increase in tourist demand in Alpine regions.

In Alpine regions, tourist demand is determined by many factors such as climate, landscape, access to lakes, prices, public indoor facilities, etc. A couple of studies have shown that climate conditions are amongst the dominant factors affecting choice of destination (Hamilton and Lau 2006; Lise and Tol 2002). However, no study has investigated the impact of weather conditions in lowland regions (the push factor) on the tourism destinations. In this paper, we quantify the observed impact of hot summer air temperature on tourism demand in the Swiss Alps by analysing the relationship between temperature and overnight stays at 40 Alpine resorts. Several temperature thresholds were tested to detect whether there was a relationship with summer tourism. We restricted our study to domestic tourism in order to detect whether hot temperatures at lower elevations were resulting in lowland residents spending time at higher elevations in the Alps.

\section{Data and methods}

We used the number of overnight stays for domestic tourism from 40 Swiss Alpine resorts (Fig. 1). The data originated from the datasets of the Federal Statistics Office 


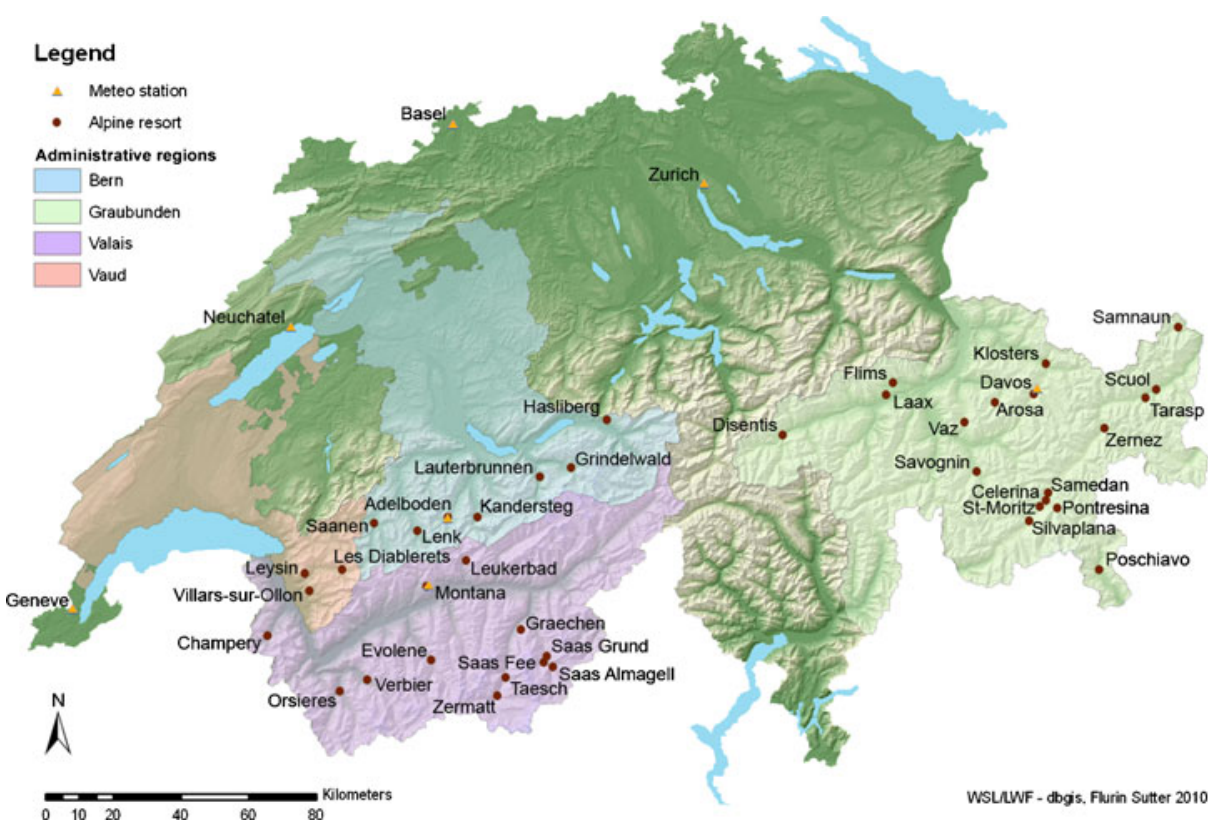

Fig. 1 Location of Alpine resorts and meteorological stations

(FSO). We used data from the four main Alpine administrative regions in the Swiss Alps: Vaud, Valais, Bern and Graubunden. In each region, we selected all Alpine ski resorts above $1000 \mathrm{~m}$ asl with a minimum of 500 beds during at least eight of the 10 available years for the period 1997 to 2007 (2004 data are missing in the FSO dataset). We limited our study to 10 years in order to have the most homogeneous data for available beds in each resort and the most homogeneous population size and behaviour in the related lowland cities. The absolute number of domestic overnight stays was averaged by the number of available beds for each month and at each of the 40 selected resorts. We removed the trends from the series.

The resulting series were correlated with temperature and sunshine duration data provided by MeteoSwiss. For both these meteorological parameters, we used several thresholds, either above an absolute ${ }^{\circ} \mathrm{C}$ value or a relative value above a percentile, as described in Table 1. The temperature values originate from the main Swiss cities at lower elevation (Geneva, Neuchatel, Basel and Zurich), primary sources for domestic tourists seeking to escape the heat of summer.

We used daily maximum temperature values for June, July and August (JJA) and computed the monthly number of days above two thresholds, i.e. higher than $25^{\circ} \mathrm{C}$ according to the common definition of a summer day (Alexander et al. 2006) used by MeteoSwiss or higher than percentile 80 of daily summer values (JJA) during the period 1997 to 2007 (without 2004), as used for instance by Rebetez (2004) for the definition of abnormally hot days. We also computed the sum of degrees per month over these two thresholds. All series were detrended.

We used relative sunshine duration from three meteorological stations at higher elevations in the Swiss Alps: Montana, $1508 \mathrm{~m}$ asl, for the regions of Valais and 
Table 1 Summary of the correlations computed between meteorological parameters and overnight stays

\begin{tabular}{|c|c|c|c|c|}
\hline \multirow{6}{*}{$\begin{array}{l}\text { Overnight } \\
\text { stays in } \\
\text { regional } \\
\text { Alpine } \\
\text { resorts }\end{array}$} & Maximum temperature & \multicolumn{3}{|c|}{ Relative sunshine duration } \\
\hline & Monthly nb days $>25^{\circ} \mathrm{C}$ & \multicolumn{3}{|c|}{ Monthly nb of days $>70 \%$} \\
\hline & Monthly nb days $>$ percentile 80 & \multicolumn{3}{|c|}{ Monthly nb of days $>$ percentile 80} \\
\hline & Monthly sum of degree days $>25^{\circ} \mathrm{C}$ & \multicolumn{3}{|c|}{ Monthly sum of daily percentages $>70 \%$} \\
\hline & $\begin{array}{l}\text { Monthly sum of degree days }> \\
\text { percentile } 80\end{array}$ & \multicolumn{3}{|c|}{$\begin{array}{l}\text { Monthly sum of daily percentages }> \\
\text { percentile } 80\end{array}$} \\
\hline & Mean GE, NE, BS, ZH & Montana & Adelboden & Davos \\
\hline Vaud & $\mathrm{X}$ & $\mathrm{X}$ & & \\
\hline Valais & $\mathrm{X}$ & $\mathrm{X}$ & & \\
\hline Bern & $\mathrm{X}$ & & $\mathrm{X}$ & \\
\hline Graubunden & $\mathrm{X}$ & & & $\mathrm{X}$ \\
\hline
\end{tabular}

$X$ correlation was computed. GE Geneva, NE Neuchatel, BS Basel, ZH Zurich

Vaud, Adelboden, at $1320 \mathrm{~m}$ asl, for the Bernese Alps, and Davos, at 1,590 m asl for Graubunden. Thresholds were chosen for absolute (above 70\%) and relative values (above percentile 80 ). Both the monthly number of days and the sum of daily percentages above every threshold were computed and detrended.

As potential explanatory factors for the differences observed in the resulting correlations, we also computed the average length of stay in every Alpine resort and the distance in $\mathrm{km}$ and hours between each resort and the nearest main city (Lausanne for Vaud and Valais, Bern for the Bernese Alps and Zurich for Graubunden) using www.mappy.ch. We also correlated the average length of stay with the meteorological values (both temperature and sunshine duration).

\section{Results}

Pearson's correlation coefficients for the summer months' temperature in relation to overnight stays are shown in Table 2. We combined all methods for the classification of the resorts (Table 2, right column).

Twenty-four resorts had at least one significant correlation, but there were strong differences between resorts and months. Fourteen resorts had at least one significant correlation with all four methods or thresholds for at least one month (usually June) or with at least three methods and for at least two months. Significant correlations were observed in every region, but less frequently in Graubunden: $38.9 \%$ of the resorts in Graubunden compared to $57.1 \%$ in Bern, $83.3 \%$ in Valais and $100 \%$ in Vaud. Significant correlations were observed more frequently in June (up to 15 resorts) and to a lesser extend in August (up to 10) than in July (up to 4).

The Pearson's correlation coefficients for the summer months' sunshine duration in relation to overnight stays are shown in Table 3. These results are less significant than those concerning temperature: 20 Alpine resorts had at least one positive significant correlation with sunshine duration compared to 24 with temperature. Bernese resorts had the greatest number of positive significant correlations: $71.4 \%$ of the resorts had at least one positive significant correlation compared to $66.7 \%$ for Vaud, $44.4 \%$ for Graubunden and $41.7 \%$ for Valais. 
Table 2 Pearson's correlation coefficients for the summer months' temperature

\begin{tabular}{|c|c|c|c|c|c|c|c|c|c|c|c|c|c|}
\hline \multirow[b]{2}{*}{ Alpine resorts } & \multicolumn{3}{|c|}{$\mathrm{Nb}$ days $\mathrm{Tmax}>25$} & \multicolumn{3}{|c|}{ Nb days Tmax > perc. 80} & \multicolumn{3}{|c|}{ Sum degrees Tmax $>25$} & \multicolumn{3}{|c|}{$\begin{array}{l}\text { Sum degrees Tmax } \\
>\text { perc. } 80\end{array}$} & \multirow[b]{2}{*}{ Total } \\
\hline & June & July & August & June & July & August & June & July & August & June & July & August & \\
\hline DV Villars-sur-Ollon & 0.6883 & 0.5764 & 0.5820 & 0.7306 & 0.5334 & 0.7031 & 0.7594 & 0.5434 & 0.7480 & 0.7167 & 0.4577 & 0.8085 & 9 \\
\hline BE Adelboden & 0.5092 & 0.4481 & 0.7964 & 0.5532 & 0.6986 & 0.5247 & 0.5746 & 0.6054 & 0.5670 & 0.5975 & 0.5951 & 0.4374 & 8 \\
\hline BE Kandersteg & -0.0023 & 0.8984 & 0.6268 & -0.0474 & 0.6871 & 0.7722 & 0.0111 & 0.7874 & 0.7359 & 0.0396 & 0.7195 & 0.7261 & 8 \\
\hline VS Evolene & 0.7228 & 0.3411 & 0.7572 & 0.7730 & 0.4061 & 0.7418 & 0.7897 & 0.4022 & 0.7478 & 0.8013 & 0.3420 & 0.6659 & 8 \\
\hline GR Samnaun & 0.5610 & 0.3599 & 0.3686 & 0.7358 & 0.6150 & 0.3845 & 0.7457 & 0.5565 & 0.4046 & 0.7894 & 0.6073 & 0.4490 & $\overrightarrow{7}$ \\
\hline GR Silvaplana & 0.3829 & 0.3956 & 0.4509 & 0.5648 & 0.3090 & 0.6973 & 0.5858 & 0.3485 & 0.6444 & 0.6625 & 0.4025 & 0.6364 & 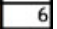 \\
\hline VS Saas Fee & 0.4854 & -0.3497 & 0.6777 & 0.5320 & -0.4795 & 0.8273 & 0.5752 & -0.3786 & 0.7789 & 0.5755 & -0.3079 & 0.7539 & 6 \\
\hline GR Arosa & 0.2650 & 0.2541 & 0.7892 & 0.4493 & 0.2793 & 0.8177 & 0.4512 & 0.2871 & 0.8772 & 0.5909 & 0.3078 & 8670 & \\
\hline $\mathrm{SSZe}$ & 0.7175 & 0.3371 & 0.4825 & 0.7752 & 0.5 & 0.4709 & 0.7770 & 0.4806 & 0.5258 & 0.7086 & 0.5104 & 5793 & $\Rightarrow$ \\
\hline GR Dis & 0.7036 & -0.3295 & 0.1823 & 0.7321 & -0.0907 & 0.0120 & 0.7125 & -0.1623 & 0.0584 & 0.6275 & -0.0773 & 0.0770 & 4 \\
\hline VD Les Diablerets & 0.5582 & -0.3145 & 0.3273 & 0.6229 & -0.3433 & 0.4415 & 0.6697 & -0.3478 & 0.4381 & 0.6381 & -0.3408 & 0.4380 & 4 \\
\hline VDL & 0.6953 & -0.0 & 0.0108 & 0.7 & -0.2129 & -0.1584 & 90 & -0.0897 & -0.1070 & & & & 4 \\
\hline VSLE & 86 & & 0.0274 & & & 0.3418 & & & 0.3221 & & & 0.4660 & 4 \\
\hline VS Orsieres & 0.6085 & -0.0041 & 0.1659 & 0.6452 & 0.2736 & 0.4078 & 0.6824 & 0.1953 & 0.3722 & 0.6281 & 0.3328 & 0.4301 & 4 \\
\hline BE Grindelwald & 0.2414 & 0.4112 & 0.4305 & 0.2738 & 0.1758 & 0.5512 & 0.3167 & 0.2660 & 0.6277 & 0.3108 & 0.1549 & 0.7142 & 7 \\
\hline $\mathrm{BEH}$ & 0.4509 & 0.1867 & 0.2180 & 0.6950 & 0.2 & 0.3398 & 0.7094 & 0.2028 & 0.3731 & 0.8076 & 57 & 0.4682 & 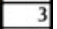 \\
\hline VSCh & & 0.3772 & 490 & & & 0.6038 & 387 & 68 & 0.6322 & 0.2678 & 0.2882 & 6902 & \\
\hline GR Pontresina & 0.5311 & 0.3002 & 0.3980 & 0.5224 & 0.1207 & 0.5248 & 0.5679 & 0.2319 & 0.4462 & 0.5666 & 0.3062 & 0.4121 & 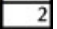 \\
\hline VS Sa & & 0.1994 & & & & & & & 26 & & 0.2416 & & 1 \\
\hline VS Ta & -0.2932 & 0.5319 & -0.1533 & -0.5591 & & 0.0012 & -0.5679 & 0.5 & -0.0290 & -0.6725 & 0.6611 & & \\
\hline GR Klosters & \begin{tabular}{|l|}
0.1098 \\
\end{tabular} & 0.6053 & 0.3229 & 0.3925 & 0.5195 & 0.3825 & 0.4009 & 0.5064 & 0.3886 & 0.4759 & 0.3124 & 0.4402 & 1 \\
\hline GR Zernez & 0.4778 & 0.3588 & 0.3654 & 0.4091 & 0.3600 & 0.5449 & 0.4075 & 0.3460 & 0.5254 & 0.3090 & 0.2925 & 0.5843 & 1 \\
\hline $\mathrm{VSCr}$ & -0.3764 & 0.6 & -0.2752 & -0.3 & 0.32 & -0.2161 & -0.3 & 0.4469 & -0.2280 & -0.2 & 0.4 & -0. & \\
\hline $\mathrm{VS} \mathrm{Gr}$ & \begin{tabular}{|l|}
0.5673 \\
\end{tabular} & -0.1372 & 0.4164 & 0.2 & -0.3 & 0.1468 & 0.2799 & -0.2727 & 0.2057 & 0.0971 & -0.2164 & 0.0995 & \\
\hline BE Lauterbrunnen & \begin{tabular}{|l|}
0.1076 \\
\end{tabular} & 0.5090 & 0.4811 & 0.1167 & 0.4413 & 0.4159 & 0.1771 & \begin{tabular}{|l|}
0.4029 \\
\end{tabular} & 0.4617 & 0.1717 & 0.1864 & 0.4718 & 0 \\
\hline BE Lenk & -0.0416 & 0.5127 & -0.1885 & -0.1868 & 0.3407 & 0.2802 & -0.1 & 0.4 & 0.2276 & -0.1407 & 0.4701 & 0.3524 & 0 \\
\hline BE Sa & 0.3256 & -0.0948 & 0.3144 & 0.3625 & -0.30 & 0.4574 & 0.4147 & -0.2 & 0.4359 & 0.4082 & -0.3097 & 0.4582 & 0 \\
\hline GR Celerina & 37 & -0.3227 & 0.2577 & 0.3335 & -0.4267 & 0.3020 & 0.3627 & -0.4187 & 0.2769 & 0.4239 & -0.4733 & 0.2567 & 0 \\
\hline GR Davos & 0.3856 & 0.4939 & -0.6721 & 0.0846 & 0.2072 & \begin{tabular}{|l|}
-0.2834 \\
\end{tabular} & 0.1063 & 0.3452 & -0.3426 & -0.0642 & 0.3402 & -0.2074 & 0 \\
\hline GR Flims & & 0.3575 & 0.2406 & & & & & & & & 0.3850 & 0.1774 & 0 \\
\hline GR La & & 0.0961 & 0.3617 & -0.0104 & 0.1123 & \begin{tabular}{|l|}
-0.0188 \\
\end{tabular} & & 0.1460 & -0.0022 & -0.0951 & 0.2751 & -0.1753 & \\
\hline GR Poschiavo & 0.4381 & 0.4135 & 0.1172 & 0.2608 & 0.2869 & 0.4323 & 0.3187 & 0.3064 & 0.3731 & 0.2173 & 0.1179 & 0.4439 & 0 \\
\hline GR Samedan & \begin{tabular}{|l|}
-0.1976 \\
\end{tabular} & -0.1041 & 0.1715 & -0.2567 & -0.1809 & 0.1029 & -0.2269 & -0.2239 & 0.0590 & -0.1887 & -0.3974 & 0.0201 & 0 \\
\hline GR Savognin & \begin{tabular}{|l|}
-0.3599 \\
\end{tabular} & 0.0472 & -0.0103 & -0.5562 & -0.1338 & 0.0731 & -0.5188 & -0.0513 & 0.0986 & -0.5510 & -0.0039 & 0.1657 & 0 \\
\hline GR Scuol & 0.3506 & 0.4474 & 01688 & 0.1626 & 0.2764 & 0.2059 & 0.2193 & 0.2965 & 0.1289 & 0.1039 & 0.1779 & 0.0475 & 1 \\
\hline GR St Moritz & \begin{tabular}{|l|}
0.1031 \\
\end{tabular} & 0.0366 & 0.1238 & 0.3704 & 0.1524 & 0.0041 & 0.4139 & 0.1428 & \begin{tabular}{|l|}
0.0509 \\
\end{tabular} & 0.4625 & 0.1677 & 0.0764 & 0 \\
\hline GR Tarasp & 0.1219 & 0.1089 & 0.3371 & -0.0868 & 0.0415 & 0.2278 & \begin{tabular}{|l|}
-0.0494 \\
\end{tabular} & 0.1136 & 0.2218 & -0.1562 & 0.2569 & 0.0665 & 0 \\
\hline GR Vaz & 0.0024 & 0.3843 & 0.2908 & -0.0886 & 0.3650 & 0.3983 & \begin{tabular}{|l|}
-0.0595 \\
\end{tabular} & 0.4304 & 0.4306 & -0.0564 & 0.4348 & 0.5238 & 4 \\
\hline VS Saas & & & & & & -0.4980 & & & & -0.1742 & & -0.3463 & 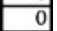 \\
\hline VS Verbier & 0.3382 & \begin{tabular}{|l|}
-0.2921 \\
\end{tabular} & 0.0532 & 0.1937 & -0.5178 & \begin{tabular}{|l|}
-0.0260 \\
\end{tabular} & 0.1958 & -0.4618 & \begin{tabular}{|l|l|} 
\\
\end{tabular} & 0.1476 & -0.4773 & -0.0829 & 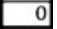 \\
\hline Total & 10 & 4 & & 12 & & & 14 & 4 & 10 & 15 & 4 & 10 & \\
\hline
\end{tabular}

Significant $(p<0.1)$ values according to Fischer's test are orange (positive) or blue (negative)

$B E$ Bern, GR Graubunden, $V D$ Vaud, $V S$ Valais

The average length of stay in every Alpine resort and the distance from the nearest important city are shown in Table 4. June has the shortest mean average length of stay (2.54 nights) compared to August (2.94) and July (3.03). The shortest average length of stay took place in June for $77.5 \%$ of the resorts, in August for $17.5 \%$ and in July for $5 \%$ of the resorts. The Bernese Alps were the only region where the shortest average length of stay was more frequent in August (57\%). The longest average length of stay took place in July for $65 \%$ of the resorts. There were no important differences between regions for this parameter.

Graubunden and East Valais resorts were located the furthest, in time and space, from the nearest main city. There was a significant negative correlation between the distance by car both in time $(R=-0.28, p=0.08)$ and space $(R=-0.30, p=0.06)$ from the nearest main city (Table 4$)$ and the relationship between temperature 
Table 3 Pearson's correlation coefficients for the summer months' sunshine duration

\begin{tabular}{|c|c|c|c|c|c|c|c|c|c|c|c|c|c|}
\hline Alpine resorts & June & July & August & June & July & August & June & July & August & June & July & August & Total \\
\hline BE Saanen & 0.5604 & 0.0712 & 0.5352 & 0.5819 & 0.6515 & 0.6855 & 0.5724 & 0.5337 & 0.6247 & 0.6002 & 0.4689 & 0.4918 & 7 \\
\hline GR Arosa & -0.5893 & 0.3588 & 0.6248 & -0.5652 & 0.7260 & 0.6854 & -0.5455 & 0.4908 & 0.6280 & -0.5333 & 0.5993 & 0.4251 & 5 \\
\hline GR Poschiavo & 0.1767 & 0.5542 & 0.4143 & 0.4656 & -0.0159 & 0.6260 & 0.4166 & 0.3048 & 0.6156 & 0.6342 & -0.1271 & 0.6708 & 5 \\
\hline BE Hasliberg & 0.4736 & 0.6652 & 0.3990 & 0.4494 & 0.5794 & 0.6568 & 0.4825 & 0.5251 & 0.5506 & 0.4280 & 0.0418 & 0.4858 & 4 \\
\hline GR Pontresina & -0.3223 & 0.4089 & 0.6670 & -0.1182 & 0.4395 & 0.7230 & -0.1303 & 0.5032 & 0.7777 & -0.0290 & 0.2791 & 0.8656 & 4 \\
\hline VS Leukerbad & 0.1788 & 0.5582 & 0.1944 & 0.3780 & 0.6023 & 0.5479 & 0.2982 & 0.6992 & 0.3547 & 0.3261 & 0.5680 & 0.3552 & 4 \\
\hline \begin{tabular}{|l} 
VS Saas Fee \\
\end{tabular} & \begin{tabular}{|l|}
-0.1868 \\
\end{tabular} & \begin{tabular}{|l|}
-0.4827 \\
\end{tabular} & 0.6994 & 0.0433 & 0.0195 & 0.7474 & \begin{tabular}{|l|}
-0.0556 \\
\end{tabular} & -0.2507 & 0.7489 & 0.0973 & -0.1835 & 0.8059 & 4 \\
\hline GR Scuol & 0.2081 & 0.6340 & 0.4480 & 0.4263 & 0.2876 & 0.4276 & 0.3783 & 0.5576 & 0.4883 & 0.5193 & 0.0415 & 0.5665 & 3 \\
\hline GR Zernez & -0.0913 & 0.0904 & 0.5337 & -0.1312 & -0.3240 & 0.7344 & -0.1276 & -0.1168 & 0.7405 & -0.3584 & -0.3758 & 0.7782 & 3 \\
\hline GR Flims & 0.4389 & 0.3570 & 0.1736 & 0.5877 & 0.0827 & 0.3264 & 0.5102 & 0.2549 & 0.1988 & 0.5824 & -0.0101 & 0.1051 & 2 \\
\hline BE Adelboden & 0.4233 & 0.5212 & 0.6392 & 0.2287 & -0.0249 & 0.4360 & 0.3634 & 0.0072 & 0.4074 & 0.3005 & -0.3050 & 0.2355 & 1 \\
\hline VD Les Diablerets & 0.0988 & 0.1198 & 0.3092 & 0.2739 & 0.5899 & 0.4911 & 0.2113 & 0.4179 & 0.3178 & 0.2593 & 0.3382 & 0.2699 & 1 \\
\hline BE Grindelwald & 0.4806 & 0.4112 & 0.3237 & 0.3737 & 0.4717 & 0.3800 & 0.3534 & 0.2885 & 0.3598 & 0.3436 & -0.0971 & 0.2900 & 0 \\
\hline BE Lenk & -0.4856 & 0.3547 & -0.1274 & -0.4589 & 0.3873 & -0.0207 & -0.5477 & 0.3612 & 0.0583 & -0.4941 & 0.2632 & 0.2515 & 0 \\
\hline GR Celerina & -0.0794 & -0.0445 & 0.3760 & 0.0072 & 0.4010 & 0.4285 & 0.0133 & 0.2391 & 0.4500 & 0.0860 & 0.5124 & 0.4482 & 0 \\
\hline GR Davos & -0.3000 & 0.4297 & -0.4050 & -0.1617 & -0.0055 & -0.3425 & -0.2516 & 0.3529 & -0.2713 & -0.2082 & -0.0371 & -0.1424 & 0 \\
\hline GR Disentis & 0.4541 & -0.6283 & -0.0627 & 0.3578 & -0.4764 & -0.0152 & 0.3636 & -0.6515 & \begin{tabular}{|l|}
-0.0666 \\
\end{tabular} & 0.0164 & -0.3132 & -0.0815 & 0 \\
\hline GR Laax & -0.2647 & -0.1903 & 0.1518 & -0.1655 & -0.1682 & -0.0368 & -0.2161 & -0.0614 & -0.0605 & -0.2257 & -0.1206 & -0.1226 & 0 \\
\hline GR Samedan & -0.8634 & 0.0437 & 0.3671 & -0.7779 & -0.0657 & 0.3770 & -0.8128 & -0.0473 & 0.4391 & \begin{tabular}{|l|}
-0.6472 \\
\end{tabular} & -0.0549 & 0.5231 & 0 \\
\hline GR Samnaun & 0.1829 & 0.1005 & 0.2687 & 0.1914 & 0.1271 & 0.4208 & 0.2541 & -0.0007 & 0.3480 & 0.0980 & 0.0346 & 0.3102 & 0 \\
\hline GR Savognin & -0.6580 & 0.1521 & 0.1344 & -0.5318 & 0.1942 & 0.2491 & -0.6051 & 0.2652 & 0.2228 & -0.3525 & 0.1885 & 0.1752 & 0 \\
\hline VS Orsieres & 0.2939 & 0.0127 & 0.2225 & 0.4527 & -0.2430 & 0.5302 & 0.4576 & -0.1835 & 0.3611 & 0.4076 & -0.2396 & 0.3426 & 0 \\
\hline VS Saas Almagell & -0.2999 & 0.1832 & -0.6208 & -0.0408 & 0.2489 & -0.7749 & -0.1066 & 0.1625 & \begin{tabular}{|l|}
-0.7616 \\
\end{tabular} & 0.0045 & -0.2127 & -0.7799 & 0 \\
\hline VS Taesch & -0.4416 & 0.3729 & 0.1080 & -0.4366 & -0.3902 & 0.3354 & -0.5041 & 0.0253 & 0.2573 & -0.3567 & -0.3831 & 0.2108 & 0 \\
\hline VS Verbier & 0.2001 & -0.5419 & 0.4185 & 0.3007 & -0.1504 & 0.5312 & 0.3194 & -0.4051 & 0.5138 & 0.3330 & 0.2121 & 0.4613 & 0 \\
\hline Total & 2 & 6 & 8 & 4 & 9 & 12 & 3 & 6 & 11 & 4 & 3 & 8 & \\
\hline
\end{tabular}

Significant $(p<0.1)$ values according to Fischer's test are orange (positive) or blue (negative)

$B E$ Bern, GR Graubunden, $V D$ Vaud, $V S$ Valais

and overnight stays (Table 2, right column). But the correlation between the mean monthly average length of stay in June-July-August (JJA) and the relationship between temperature and overnight stays is not significant $(R=-0.20, p=0.22)$. The results of the multiple regression analysis show that there is no significant correlation between the distance (in time or space) and the mean length of stay in JJA (Table 5).

\section{Discussion}

Our results show that there is an impact of high temperature values at lower elevation on the number of overnight stays made by domestic tourists in the Swiss Alps: 24 resorts revealed a correlation between the number of hot days at lower elevation and the number of overnight stays in the mountains. The correlation with temperature was stronger than with sunshine duration (20 resorts) despite the well-known positive correlation between temperature and sunny days in summer in the Swiss Alps: 
Table 4 Average length of stay (number of days) and distance ( $\mathrm{km}$ and hours) from the nearest main city

\begin{tabular}{|c|c|c|c|c|c|c|}
\hline \multirow[t]{2}{*}{ Alpine resorts } & \multicolumn{4}{|c|}{$\begin{array}{l}\text { Average length of stay } \\
\text { (no. of days) }\end{array}$} & \multicolumn{2}{|c|}{$\begin{array}{l}\text { Distance } \\
\text { (km and hours) }\end{array}$} \\
\hline & June & July & August & Mean JJA & Length & Time \\
\hline VD Leysin & 2.72 & 4.27 & 3.66 & 3.55 & 61.34 & $00: 53$ \\
\hline VD Villars-sur-Ollon & 2.12 & 2.65 & 2.55 & 2.44 & 59.18 & $00: 55$ \\
\hline BE Kandersteg & 2.42 & 2.64 & 2.46 & 2.51 & 66.66 & $00: 57$ \\
\hline VD Les Diablerets & 2.43 & 4.54 & 3.56 & 3.51 & 66.34 & 00:58 \\
\hline BE Kanderstag & 2.05 & 1.99 & 1.95 & 1.99 & 63.47 & 00:59 \\
\hline VS Champery & 2.23 & 2.87 & 2.28 & 2.46 & 63.99 & 00:59 \\
\hline BE Adelboden & 3.34 & 3.34 & 3.19 & 3.29 & 67.04 & 01:08 \\
\hline VS Verbier & 1.63 & 2.14 & 2.01 & 1.93 & 91.73 & $01: 10$ \\
\hline BE Grindelwald & 1.91 & 2.15 & 2.04 & 2.03 & 53.13 & 01:11 \\
\hline BE Hasilberg & 4.42 & 4.47 & 4.24 & 4.38 & 88.26 & 01:14 \\
\hline VS Orsieres & 2.07 & 2.24 & 2.17 & 2.16 & 91.17 & 01:14 \\
\hline GR Films & 2.88 & 3.60 & 3.23 & 3.23 & 137.68 & 01:33 \\
\hline VS Crans-Montana & 3.60 & 4.56 & 4.46 & 4.21 & 124.93 & $0: 133$ \\
\hline VS Evolone & 1.74 & 1.96 & 1.90 & 1.87 & 119.97 & 01:35 \\
\hline GR Laax & 1.99 & 2.77 & 2.74 & 2.50 & 142.24 & 01:37 \\
\hline VS Leukerbad & 3.83 & 3.49 & 3.37 & 3.56 & 115.53 & 01:39 \\
\hline GR Klosters & 2.74 & 2.80 & 3.08 & 2.88 & 135.55 & 01:42 \\
\hline GR Vaz & 2.73 & 3.42 & 3.26 & 3.14 & 141.78 & $01: 45$ \\
\hline BE Saanen & 2.71 & 2.75 & 2.62 & 2.69 & 83.87 & 01:50 \\
\hline GR Savognin & 2.13 & 2.22 & 2.11 & 2.16 & 163.98 & 01:52 \\
\hline BE Lenk & 3.59 & 3.10 & 3.32 & 3.34 & 83.29 & $01: 53$ \\
\hline GR Davos & 3.08 & 3.81 & 3.88 & 3.59 & 147.43 & 01:53 \\
\hline GR Disentis & 1.86 & 1.97 & 1.93 & 1.92 & 141.64 & 01:56 \\
\hline GR Arosa & 2.73 & 3.81 & 3.79 & 3.44 & 150.68 & 01:57 \\
\hline GR Zernez & 1.98 & 1.96 & 2.06 & 2.00 & 167.62 & 02:08 \\
\hline VS Saas Grund & 2.97 & 3.12 & 3.45 & 3.18 & 162.05 & 02:09 \\
\hline VS Saas Fee & 2.37 & 3.10 & 3.14 & 2.87 & 164.48 & $02: 12$ \\
\hline VS Graechen & 2.58 & 3.63 & 3.52 & 3.24 & 162.74 & $02: 13$ \\
\hline VS Saas Almagell & 3.33 & 4.17 & 4.12 & 3.87 & 165.78 & $02: 15$ \\
\hline GR Scuol & 2.85 & 2.87 & 2.84 & 2.85 & 179.54 & $02: 15$ \\
\hline GR Tarasp & 3.27 & 3.89 & 3.64 & 3.60 & 179.54 & $02: 18$ \\
\hline VS Taesch & 1.61 & 1.75 & 1.87 & 1.74 & 169.41 & $02: 21$ \\
\hline GR Silvaplano & 2.24 & 3.15 & 3.00 & 2.80 & 197.86 & $02: 26$ \\
\hline GR Samedan & 2.10 & 2.71 & 2.78 & 2.53 & 195.46 & $02: 33$ \\
\hline GR St Moritz & 2.11 & 2.95 & 3.01 & 2.69 & 203.92 & $02: 33$ \\
\hline GR Celerina & 2.85 & 3.36 & 3.44 & 3.22 & 197.62 & $02: 36$ \\
\hline VS Zermatt & 1.95 & 2.56 & 2.56 & 2.36 & 175.38 & $02: 37$ \\
\hline GR Pontrresina & 2.79 & 3.99 & 3.77 & 3.52 & 199.65 & $02: 38$ \\
\hline GR Samnaun & 1.68 & 2.35 & 2.43 & 2.15 & 215.95 & $02: 53$ \\
\hline GR Poschiavo & 2.54 & 2.23 & 2.16 & 2.18 & 232.54 & 03:08 \\
\hline Mean & 2.54 & 3.03 & 2.94 & 2.84 & & \\
\hline
\end{tabular}

$B E$ Bern, GR Graubunden, $V D$ Vaud, $V S$ Valais

contrary to winter-time conditions, at higher elevation, a summer rainy day is colder on average than a sunny day (Rebetez 1996). The number of overnight stays in the Alpine resorts of Vaud, Valais and Bern had a higher correlation with temperature 
Table 5 Multiple regression analysis with Anova for the relationship between temperature and overnight stays (Table 2, right column), distance in space and time from the nearest city and mean length of stay in June-July-August (JJA)

\begin{tabular}{|c|c|c|c|c|c|}
\hline & $d f$ & Sum sq & Mean sq & $F$ value & $\operatorname{Pr}(>\mathrm{F})$ \\
\hline Distance space & 1 & 27.559 & 27.559 & 3.6821 & $0.0625 *$ \\
\hline Residuals & 38 & 284.42 & 7.485 & & \\
\hline Distance time & 1 & 23.956 & 23.956 & 3.1606 & $0.0834 *$ \\
\hline Residuals & 38 & 288.02 & 7.579 & & \\
\hline Mean length of stay JJA & 1 & 12.059 & 12.059 & 1.5279 & 0.224 \\
\hline Residuals & 38 & 299.92 & 7.893 & & \\
\hline Distance space & 1 & 27.559 & 27.559 & 3.7019 & $0.0623^{*}$ \\
\hline Mean length of stay JJA & 1 & 13.264 & 13.264 & 1.7817 & 0.1903 \\
\hline Distance space/mean length of stay JJA & 1 & 3.149 & 3.149 & 0.423 & 0.5196 \\
\hline Residuals & 36 & 268 & 7.445 & & \\
\hline Distance time & 1 & 23.956 & 23.956 & 3.2082 & $0.0817 *$ \\
\hline Mean length of stay JJA & 1 & 13.837 & 13.837 & 1.8531 & 0.1819 \\
\hline Distance time: mean length of stay JJA & 1 & 5.367 & 5.367 & 0.7187 & 0.4022 \\
\hline Residuals & 36 & 268.82 & 7.467 & & \\
\hline
\end{tabular}

*Significant at $p<0.1$

compared to Graubunden, where the correlation with sunshine duration was stronger than with temperature. This can be explained by the distance to the nearest main city. The reaction to hot summer temperatures was highest in Vaud, where the alpine tourist destinations are closest to the nearest main cities, and lowest in Graubunden, where the distance to the main cities is greatest. A possible explanation for this is that when people take a last minute decision to go to the mountains because of hot temperatures they prefer to go to nearby locations. There are some exceptions, such as Samnaun or Silvaplana, that are situated far away from the main cities but correlate well with hot temperatures. The differences which appeared between some nearby resorts such as Lenk and Kandersteg probably depend on factors other than the distance to the nearest main cities. For all these resorts, further studies, including multifactorial analyses (marketing done by the resort, public infrastructure in the resorts such as swimming pools, special seasonal offers, etc.) could explain specific behaviours.

The significant negative correlation between the distance by car (both in time and space) from the nearest main city and the relationship between temperature and overnight stays suggests that the shorter the distance, the more likely the tourists are to visit the resorts during hot temperatures.

The stronger correlation observed in June and August compared to July is probably due to school holidays and to the peak domestic tourist demand in summer, which takes place from the beginning of July until mid-August. This is normally the hottest time of the year, when long stays are planned well in advance, independent of the weather. The average length of stay was shortest in June, which suggests that occupancy may have been determined more by short-term decisions. In order to confirm this hypothesis, it would be necessary to know the reservation dates of the stays, but these data unfortunately do not exist. August has a slightly shorter average length of stay than July. This may be because school holidays normally end in early or mid-August. The stronger correlation with temperature in August 
compared to July may be because overnight stays are again organized on a shortterm basis.

\section{Conclusion}

Our results demonstrate a correlation between domestic tourist demand and hot summer temperatures in the Swiss Alps. Particularly during June and, to a lesser extent, August, there is a correlation between the number of nights spent in mountain resorts and hot temperatures at lower elevations, the origin of most domestic tourists.

Alpine resorts nearest to major cities had stronger correlations with hot summer temperatures, and this may be because reactions to hot episodes take place on a short-term basis, with the result that nearby mountain resorts are chosen.

Our results suggest that summer occupancy in Alpine tourist resorts may benefit from hotter temperatures at lower elevations. Domestic tourists appear to react to hot days by spending more nights in hotels in the mountains. If heat waves increase in frequency and intensity, it is possible that domestic tourists will choose to go to mountain resorts more frequently or for longer periods. The observed patterns may affect tourists from larger areas if the summer heat experienced in 2003 or 2006 becomes more frequent.

The results concerning the impact of hot temperatures and sunshine duration on domestic overnight stays show that further studies could include multifactorial analyses (marketing done by the resort, public infrastructure of the resorts such as swimming pools, special offers, etc.) in order to measure the impact of each factor on the overnight stays. Variables other than overnight stays (restaurant covers, cable car use, etc.) could also be included in order to quantify the impact of hot temperature episodes on day trippers.

Acknowledgements This work was supported in part by Canton Vaud, Switzerland, and by the Forest Investigation Programme, a joint project between the Swiss Federal Office for the Environment and the WSL Swiss Federal Institute for Forest, Snow and Landscape Research. We are grateful to MeteoSwiss and to the Swiss Federal Statistical Office for providing the data, to Flurin Suter for drawing the map and to John Innes and Christophe Clivaz for their useful comments and suggestions.

\section{References}

Alexander LV, Zhang X, Peterson TC, Caesar J, Gleason B, Tank A, Haylock M, Collins D, Trewin B, Rahimzadeh F, Tagipour A, Kumar KR, Revadekar J, Griffiths G, Vincent L, Stephenson DB, Burn J, Aguilar E, Brunet M, Taylor M, New M, Zhai P, Rusticucci M, Vazquez-Aguirre JL (2006) Global observed changes in daily climate extremes of temperature and precipitation. J Geophys Res-Atmos 111:D05109. doi:05110.01029/02005jd006290

Amelung B, Viner D (2006) Mediterranean tourism: exploring the future with the Tourism Climatic Index. J Sustain Tour 14:349-366

Ceron J-P, Dubois G (2000) Tourisme et changement climatique. In: Impacts et potentiels du changement climatique en France au XXIème Siècle, 2nd edn. Premier Ministre, Ministère de l'Aménagement du Territorie et de l'Environnement, pp 104-111

Della-Marta PM, Haylock MR, Luterbacher J, Wanner H (2007a) Doubled length of western European summer heat waves since 1880. J Geophys Res-Atmos 112:D15103. doi:15110.11029/ 12007JD008510 
Della-Marta PM, Luterbacher J, von Weissenfluh H, Xoplaki E, Brunet M, Wanner H (2007b) Summer heat waves over western Europe 1880-2003, their relationship to large-scale forcings and predictability. Clim Dyn 29:251-275

Elsasser H, Messerli P (2001) The vulnerability of the snow industry in the Swiss Alps. MT Res Dev 21:335-339

Elsasser H, Buerki R (2002) Climate change as a threat to tourism in the Alps. Clim Res 20:253-257

Hamilton JM, Lau MA (2006) The role of climate information in tourist destination choice decision making. In: Gössling S, Hall CM (eds) Tourism and global environmental change: ecological, social, economic and political interrelationships. Routledge, London, pp 229-225

Hamilton JM, Maddison DJ, Tol RSJ (2005) Climate change and international tourism: a simulation study. Glob Environ Change Part A 15:253-266

Hantel M, Hirtl-Wielke LM (2007) Sensitivity of Alpine snow cover to European temperature. Int J Climatol 27:1265-1275

IPCC (2007a) Climate change 2007. The scientific basis. IPCC, Cambridge, p 994

IPCC (2007b) Europe. Climate change 2007: impacts, adaptation and vulnerability. IPCC, Cambridge, pp 541-580

Koenig U, Abegg B (1997) Impacts of climate change on winter tourism in the Swiss Alps. J Sustain Tour 5:46-58

Lise W, Tol RSJ (2002) Impact of climate on tourist demand. Clim Change 55:429-449

Marty C (2008) Regime shift of snow days in Switzerland. Geophys Res Lett 35:12501-12501

Meier R (1998) Sozioökonomische Aspekte von Klimaänderungen und Naturkatastrophen in der Schweiz. Schlussbericht NFP 3, vdf, Zürich

OcCC-ProClim (2007) Climate change and Switzerland 2050. Expected impacts on environment, society and economy. OcCC/ProClim-Forum for Climate and Global Change, Bern

OFS (2007) La statistique suisse du tourisme. OFS, Neuchâtel

Rebetez M (1996) Seasonal relationship between temperature, precipitation and snow cover in a mountainous region. Theor Appl Climatol 54:99-106

Rebetez M (2004) Summer 2003 maximum and minimum daily temperatures over a 3300 m altitudinal range in the Alps. Clim Res 27:45-50

Rebetez M, Mayer H, Dupont O, Schindler D, Gartner K, Kropp JP, Menzel A (2006) Heat and drought 2003 in Europe: a climate synthesis. Ann For Sci 63:569-577

Schaer C, Vidale PL, Luthi D, Frei C, Haberli C, Liniger MA, Appenzeller C (2004) The role of increasing temperature variability in European summer heatwaves. Nature 427:332-336

Scherrer SC, Appenzeller C (2006) Swiss Alpine snow pack variability: major patterns and links to local climate and large-scale flow. Clim Res 32:187-199

Seneviratne SI, Luthi D, Litschi M, Schaer C (2006) Land-atmosphere coupling and climate change in Europe. Nature 443:205-209

Stott PA, Stone DA, Allen MR (2004) Human contribution to the European heatwave of 2003. Nature 432:610-614

STV-FST (2008) Swiss tourism in figures. STV-FST, Bern

WEF (2007) The travel \& tourism competitiveness report 2007. Furthering the process of economic development. World Economic Forum, Geneva

WEF (2008) The travel \& tourism competitiveness report 2008. Balancing economic development and environmental sustainability. World Economic Forum, Geneva

WEF (2009) The travel \& tourism competitiveness report 2009. Managing in a time of turbulence. World Economic Forum, Geneva

Wielke LM, Haimberger L, Hantel M (2004) Snow cover duration in Switzerland compared to Austria. Meteorologische Zeitschrift 13:13-17 